\title{
Optimization, Control and Shape Design of an Arterial Bypass
}

\author{
Gianluigi Rozza* \\ CMCS - Modelling and Scientific Computing, \\ Institute of Analysis and Scientific Computing (IACS), \\ EPFL, École Polytechnique Fédérale de Lausanne, \\ CH-1015, Lausanne, Switzerland. \\ E-mail:Gianluigi.Rozza@epfl.ch
}

\begin{abstract}
SUMMARY
We present multi-level geometrical approaches in the study of aorto-coronaric bypass anastomoses configurations. The theory of optimal control based on adjoint formulation is applied in order to optimize the shape of the incoming branch of the bypass (the toe) into the coronary. At this level two possible options are available in shape design: one implements local boundary variations in computational domain, the other is based on a linearized design in a suitable reference domain through the theory of small perturbations. At a coarser level, reduced basis methodologies based on parametrized partial differential equations are developed to provide (a) a sensitivity analysis for geometrical quantities of interest in bypass configurations and (b) rapid and reliable prediction of integral functional outputs. The aim is (i) to provide design indications for arterial surgery in the perspective of future development for prosthetic bypasses, (ii) to develop multi-level numerical methods for optimization and shape design by optimal control, and (iii) to provide an input-output relationship led by models with lower complexity and computational costs. We have numerically investigated a reduced model based on Stokes equations and a vorticity cost functional (to be minimized) in the down-field zone of bypass. In local shape design procedure a Taylor like patch has been found. A feedback procedure with Navier-Stokes fluid model is proposed based on the analysis of wall shear stress and its related indexes of interest. Copyright (c) 2004 John Wiley \& Sons, Ltd.

KEY WORDs: Optimal Control, Shape Design, Small Perturbations Theory, Flow Control, Parametrized PDEs, Generalized Stokes Problem, Reduced Basis Methods, Arterial Bypass Optimization.
\end{abstract}

\section{FRAMEWORK: CFD in HAEMODYNAMICS}

When a coronary artery is affected by a stenosis, the heart muscle cannot be properly oxygenated through blood. Aorto-coronaric anastomosis restores the oxygen amount through

\footnotetext{
*Correspondence to: Gianluigi Rozza, EPFL, IACS-CMCS, CH-1015 Lausanne, Switzerland.

Contract/grant sponsor: EU-RTN, European Union Research Training Network, HaeMOdel Project; contract/grant number: HPRN-CT-2002-002760
} 
a bypass surgery downstream an occlusion. At present, different kind and shape of aortocoronaric bypass anastomoses are available and, consequently, different surgery procedures are used to set up a bypass. A bypass can be made up either by organic material (e.g. the saphena vein taken from patient's legs or the mammary artery) or by prosthetic material. Prosthetic bypasses are less invasive. They may feature very different shapes for bypass anastomoses, such as, e.g., cuffed arteriovenous access grafts.

Mathematical modelling and numerical simulation of physiological flows allow better understanding of phenomena involved in coronary diseases (see References [10] and [11]). Improvement in the understanding of the genesis of coronary diseases is very important as it allows the reduction of surgical and post-surgical failures. It may also suggest new means in bypass surgical procedures as well as with less invasive methods to devise new shape in bypass configuration (see Reference [6] for an introduction to optimal design for arterial bypass anastomosis).

A geometrical multi-level approach. In this work, the background provided by mathematical modelling and numerical simulation has led us to apply the Optimal Control theory of systems governed by partial differential equations (PDEs) with the aim of optimizing the (full) configuration and the (local) shape of a simplified bypass model. In support to this aim at macro-geometrical level efficient schemes for reduced-basis methodology [7] applied to parametrized partial differential equations $\left(P^{2} D E s\right)$ are being used to provide useful and quick indications (outputs) in a repetitive design environment as shape design requires. With the reduced basis approach also a sensitivity analysis of the initial configuration and a study of important geometrical quantities in bypass is under investigation (see References [13] for an introduction and [5] for details). Fig. 1 clarifies our geometrical double-level of interest for bypass design.

A double control approach. At micro-geometrical level optimal control of one (or several) aspect of the problem entails the minimization of a cost functional which describes physical quantities involved in the specific problem. The problem is related both with optimal shape design (see B.Mohammadi, O.Pironneau [4]) and flow control (involved in the observation of the evolving system and in cost functionals (such as vorticity or wall shear stress)). Optimization process is carried out by a control function used as parameter in modelling the shape of the domain. At this level a double control approach has been used: in the former case control function is used to define directly the boundary shape (local boundary variation method) in the true domain (see Reference [9]); in the latter case control function is used to define the mapping transformation from the reference domain to the true one. In this case the design problem becomes an optimal control problem on coefficients and the analysis is based on small perturbation theory (see Reference [1]). In both cases the adjoint approach proposed by J.L. Lions [3] to get cost functionals gradient in problem with distributed or boundary control and observation has been developed. In the functional optimization process a descent gradient-type method is used. Numerical approximation is based on Galerkin-Finite Element Method (see for example Reference [12]).

Results. At the end of a first investigation stage, which has been preliminary reported in [9], based on optimal design by local boundary variation, a cuffed bypass is found with a shape which resembles the Taylor arterial patch [2]. Results reported in Sec. 4, based on the multilevel and double control approaches, go in the same direction. The effect of the shape obtained is to reduce gradually the average velocity of the blood as it approaches the distal anastomosis, since the cross-sectional area of the bypass is steadily becoming larger. This prevents the 
sudden deceleration experienced in the conventional model with the fluid returning to the host vessel. The blood is guided more smoothly through the vessel thanks to the gradually changing geometry. Consequently there is a smooth reduction of the momentum of the blood while approaching the junction. Flow disturbances are abated, undesirable flow separation at the toe of the bypass diminished. Vorticity reduction by the optimization process is quite substantial. A feedback procedure has then been implemented by solving the unsteady NavierStokes equations in the original configuration as well as in the final configuration obtained after applying the optimal shape design process on the simplified model. The quadratic functional used at this step keeps into consideration wall shear stress variations in time and along the vascular wall we are modelling by shape design. A reduction of $25 \%$ in wall shear stress spatial and temporal oscillations has been guaranteed.

Development Guidelines. Optimal control and shape optimization applied to fully unsteady incompressible Stokes and Navier-Stokes equations and the setting of the problem in a three-dimensional geometry will provide more realistic design indications concerning surgical prosthesis realizations. Theoretical investigation based on perturbation theory analysis and linearized shape design is providing results on existence and uniqueness of solution and about well-posedness of the problem, and will permit us to better understand the problem from a theoretical point of view. Reduced-basis methodology approximation is going to provide not only high computational savings but also a methodological pre-process to detect the essential feature of the optimization process itself (such as a sensitivity analysis). The ultimate goal is to build an input-output relationship $s_{i}=F_{i}\left(\mu_{k}\right)$ with different models characterized by an increasing degree of complexity, where $s_{i}$ are outputs of interest (design quantities and fluid mechanics indexes) and $\mu_{k}$ inputs (typically geometrical quantities).

\section{CONTROL and SHAPE DESIGN: A DOUBLE APPROACH}

The Stokes equations in a two-dimensional computational domain $\Omega$ with velocity vector $\mathbf{u}=\{u, v\}$ and pressure $p$ read:

$$
\left\{\begin{array}{l}
-\nu \Delta \mathbf{u}+\nabla p=0 \text { in } \Omega \subset \mathbb{R}^{2}, \\
\nabla \cdot \mathbf{u}=0 \text { in } \Omega, \\
\mathbf{u}=0 \text { on } \Gamma_{w}, \mathbf{u}=g_{\text {in }} \text { on } \Gamma_{\text {in }}, T \cdot \hat{n}=0 \text { on } \Gamma_{\text {out }},
\end{array}\right.
$$

where $\hat{n}$ is normal unit vector on the boundary $\partial \Omega$. The latter is partitioned in three components: $\Gamma_{i n}$ is the inflow boundary, where a Hagen-Poiseuille's velocity profile $g_{i n}$ is imposed, $\Gamma_{\text {out }}$ is the arterial outflow boundary, with a free-stress Neumann-type condition on stress tensor $T$, and $\Gamma_{w}$ is the boundary corresponding to the arterial wall, the stenosed artery portion and the incoming branch of bypass with no-slip conditions imposed; Fig. 1 represents schematically the computational geometry and the symbols used.

Velocity values at the inflow are chosen so that the Reynolds number has order $10^{3}$. Blood kinematic viscosity $\nu$ is $4 \cdot 10^{-6} \mathrm{~m}^{2} \mathrm{~s}^{-1}$ [10].

In this first approach the control $w$ represents the shape of $\Gamma_{c}$ : a part of $\Gamma_{w}$ (typically the upper part of the incoming branch), made up of $M$ branches $\Gamma_{c}^{j}(w)=\overline{\Gamma_{c}^{j}}+w^{j}$, where $w^{j}$ is the control variable, the curves $\overline{\Gamma_{c}^{j}}$ are shape functions (polynomials). The control shape function $w^{j}$ changes of a quantity $\delta w^{j}$ during the optimization process. At the k-th iteration we have: $w_{k}^{j}(s)=\sum_{m=0}^{k-1}\left(\delta w_{m}^{j}\right)$. 

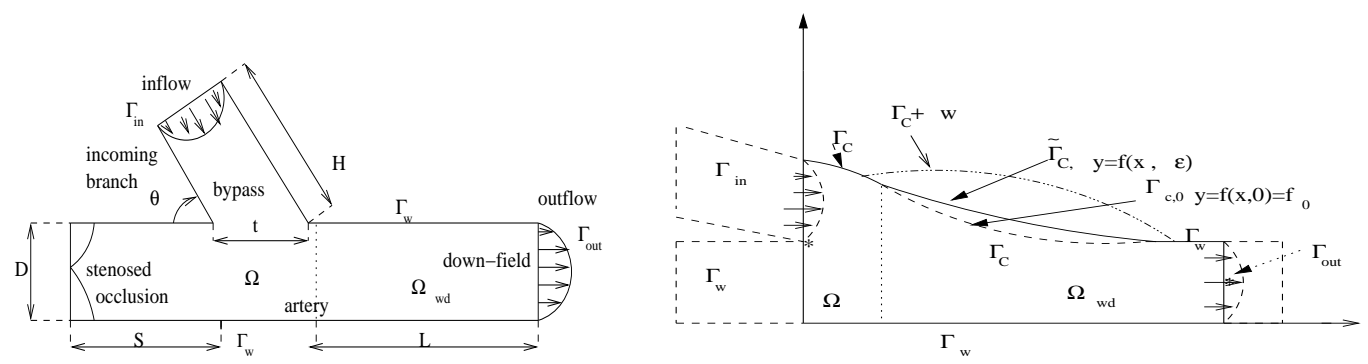

Figure 1. Bypass schemes: macro geometrical (left) and local configurations (right).

The observation on the system. We consider vorticity as distributed observation (flow control and shape optimization combined problems) in the down-field zone $\Omega_{w d}$ of the incoming branch of the bypass, defined as $\nabla \times \mathbf{u}=\frac{\partial v}{\partial x}-\frac{\partial u}{\partial y}$ and we control the system by minimizing the functional: $J(w)=\int_{\Omega_{w d}}|\nabla \times \mathbf{u}|^{2} d \Omega$. During the optimization iterative process we must solve the following adjoint problem, to estimate the cost functional gradient $J^{\prime}(w)$ :

$$
\left\{\begin{array}{l}
-\nu \Delta \mathbf{q}+\nabla \pi=\nabla \times \nabla \times\left.\mathbf{u}\right|_{\Omega_{w d}} \text { in } \Omega \\
\nabla \cdot \mathbf{q}=0 \text { in } \Omega, \mathbf{q}=0 \text { on } \partial \Omega
\end{array}\right.
$$

where $\mathbf{q}$ and $\pi$ denote the adjoint velocity and pressure, respectively. The feedback procedure based on unsteady Navier-Stokes applied on the two different configurations is based on the following cost functional:

$$
J_{\tau}=\operatorname{mean}_{0 \leq t \leq T} \Sigma(t)=\frac{1}{T} \int_{0}^{T} \Sigma(t) d t=\frac{1}{T} \int_{0}^{T} \int_{\Gamma_{w}}\left(\frac{\partial}{\partial t} \tau_{w}(t)\right)^{2} d \Gamma d t
$$

which is the $L^{2}$ norm of the rate of the wall shear stress $\left(\tau_{w}(t)\right)$. See for details [9].

Small perturbations. A second approach to local shape design is based on a map from the real domain $\Omega$ to a (rectangular) reference one $\tilde{\Omega}$ using a variable transformation $\tilde{x}=x, \tilde{y}=\frac{1}{f(x, \varepsilon)} y$, where $f(x, \varepsilon)$ represents the upper shape and can be developed as $f(x, \varepsilon)=$ $f_{0}(x)+\varepsilon f_{1}(x)+\varepsilon^{2} f_{2}(x)+\ldots$, being $f_{0}(x)$ the unperturbed shape. Assuming that problem (1) has a solution $\mathbf{u}, p$ that is infinitely differentiable with respect to $\varepsilon: \mathbf{u}=\mathbf{u}_{\mathbf{0}}+\varepsilon \mathbf{u}_{\mathbf{1}}+\ldots$, and $p=p_{0}+\varepsilon p_{1}+\ldots$, and using small perturbation techniques we can derive the equations for $\mathbf{u}_{\mathbf{k}}, p_{k}$ starting from (1), after mapping $\Omega$ to the reference domain. At this point we can use optimal control techniques to solve the problem for $\mathbf{u}_{\mathbf{1}}, p_{1}$ (the first corrections), the function $f_{1}(x)$ represents a perturbation in the shape $f_{0}(x)$ (weighted by $\varepsilon$ ) and is another unknown for the problem, used as control variable. Like in the first approach we use an adjoint formulation, a gradient-type method and the same functional (observation). In this case the shape design problem is transformed into an optimal control problem on the coefficients, which depend on the coordinate transformation itself. Results are shown in Fig. 3 and a detailed analysis is reported in [1]. 


\section{REDUCED BASIS TECHNIQUES FOR PRE-PROCESS}

Reduced basis techniques (see A.T.Patera et al., e.g. [7] and [8]) are being used for a preprocess applied to macro bypass configuration (Fig. 1). We need quantitative information $(s(\mu))$ about sensitivity of some geometrical quantities before applying local shape design. By choosing a certain number of geometrical parameters (bypass diameter $t$, artery diameter $D$, stenosis length $S$, graft angle $\theta$, bypass bridge height $H$ ) and a number $(N)$ of sample parameters $\boldsymbol{\mu}_{k}=\left\{t_{k}, D_{k}, S_{k}, \theta_{k}, H_{k}\right\}$ we solve our state equations in a reference domain $\tilde{\Omega}$, properly mapped by an affine geometrical transformation [13] (off-line procedures). With $N$ solutions $\mathbf{u}_{k}\left(\boldsymbol{\mu}_{k}\right), p_{k}\left(\boldsymbol{\mu}_{k}\right)$ of problem (1) we build a (reduced) basis functional space for velocity $\left(\boldsymbol{\zeta}=\left\{\mathbf{u}_{k}\left(\boldsymbol{\mu}_{k}\right), \boldsymbol{\sigma}_{k}\left(\boldsymbol{\mu}_{k}\right)\right\}\right)$ and pressure $\left(\boldsymbol{\xi}=\left\{p_{k}\left(\boldsymbol{\mu}_{k}\right)\right\}\right)$. Note that $\boldsymbol{\zeta}$ has been enriched by additional velocity $\boldsymbol{\sigma}_{k}\left(\boldsymbol{\mu}_{k}\right)$ which are the so-called Supremizer solutions. They are obtained by solving the additional problem: $\int_{\tilde{\Omega}}\left(\boldsymbol{\sigma}_{k} \cdot \mathbf{w}+\nabla \boldsymbol{\sigma}_{k} \cdot \nabla \mathbf{w}\right) d \Omega=\int_{\tilde{\Omega}} p_{k} \nabla \cdot \mathbf{w} d \Omega$, where both the solution $\boldsymbol{\sigma}_{k}$ and the test functions $\mathbf{w}$ belong to the Sobolev space $\left[H_{\Gamma_{w}}^{1}(\Omega)\right]^{2}$ which vanishes on $\Gamma_{w}$. These extra functions allow the spaces $\boldsymbol{\zeta}$ and $\boldsymbol{\xi}$ to satisfy an equivalent inf-sup condition [12]. Choosing a new sample $\boldsymbol{\mu}$ we can get a new (on-line) solution for our problem s.t.: $\mathbf{u}_{\mathbf{N}}=\Sigma_{k=1}^{2 N} U_{k}(\boldsymbol{\mu}) \boldsymbol{\zeta}_{k}\left(\boldsymbol{\mu}_{k}\right), p_{N}=\Sigma_{k=1}^{N} P_{k}(\boldsymbol{\mu}) \xi_{k}\left(\boldsymbol{\mu}_{k}\right)$. The components of the weights $\mathbf{U}=\left\{U_{k}\right\}$ and $\mathbf{P}=\left\{P_{k}\right\}$ are given by the solution of the reduced basis Stokes linear system:

$$
\left\{\begin{array}{l}
-\nu \sum_{j=1}^{2 N} \Psi_{i j}(\boldsymbol{\mu})\left(\int_{\tilde{\Omega}} \nabla \zeta_{i} \cdot \nabla \zeta_{j} d \Omega\right) U_{j}+\sum_{l=1}^{N} \Phi_{l i}(\boldsymbol{\mu})\left(\int_{\tilde{\Omega}} \xi_{l} \nabla \cdot \zeta_{i} d \Omega\right) P_{l}=\Theta_{i}(\boldsymbol{\mu})\left(\int_{\tilde{\Omega}} f \zeta_{i} d \Omega\right) \\
\Sigma_{j=1}^{2 N} \Phi_{l j}(\boldsymbol{\mu})\left(\int_{\tilde{\Omega}} \xi_{l} \nabla \cdot \zeta_{j} d \Omega\right) U_{j}=0,1 \leq l \leq N, 1 \leq i \leq 2 N .
\end{array}\right.
$$

$\Psi_{i j}(\boldsymbol{\mu}), \Phi_{l i}(\boldsymbol{\mu}), \Theta_{i}(\boldsymbol{\mu})$ are the affine mapping coefficients. An adaptivity procedure in choosing parameters samples to minimize error between Galerkin-Finite Element solution and the reduced basis solution has been used. A-posteriori estimates on outputs of interest $s(\boldsymbol{\mu})$ is under investigation $([5])$.

\section{SOME NUMERICAL RESULTS}

We present below some numerical results obtained applying optimal control by local boundary variation (Fig. 2) and using small perturbations techniques (Fig. 3), starting from the same configuration with a small graft angle and a cuffed upper part (as suggested by results in [9]). Shape are smoothed at the intersection with artery to guide blood and the corner disappears.
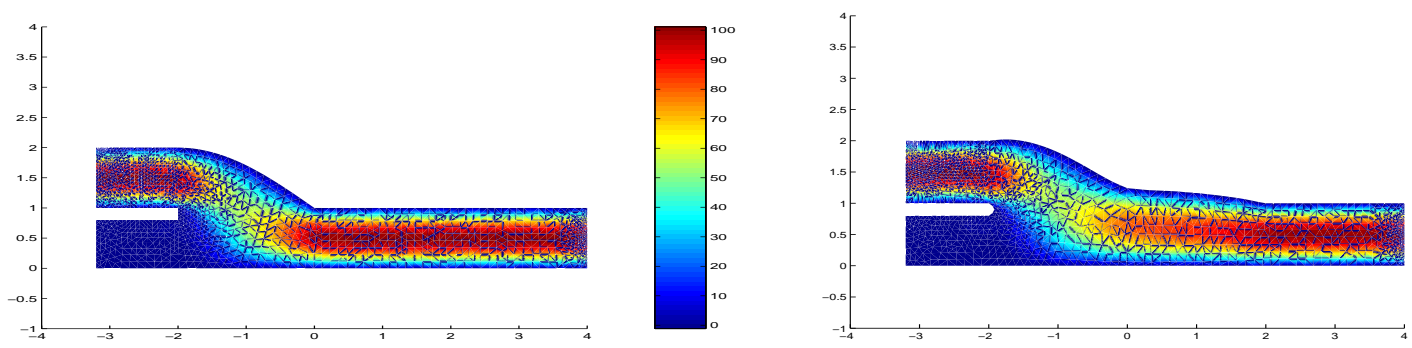

Figure 2. Bypass configuration (velocity $\left[\mathrm{ms}^{-1} \cdot 10^{-2}\right]$ ) near the incoming branch before (left) and after (local) shape optimization. 

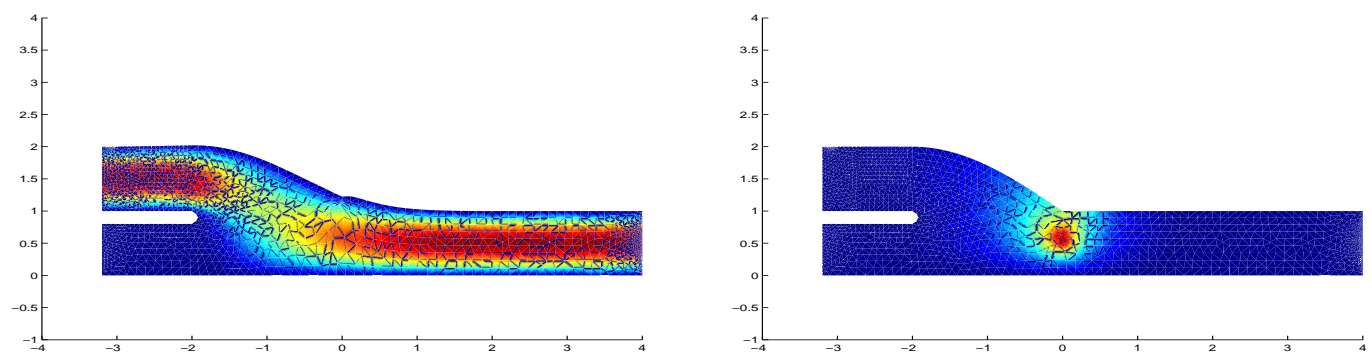

Figure 3. Linearized bypass configuration (velocity) near the incoming branch after shape design by small perturbations (right) and its adjoint solution in reference domain (right). Adjoint solution provides an indication of the most sensible zone related with our observation in the domain.

\section{ACKNOWLEDGEMENTS}

This work is being developed within the European Community's Human Potential Programme, HaeMOdel Project "Mathematical Modelling in Haemodynamics" (contract HPRN-CT-2002-002670) and the Bernoulli Center of EPFL during the special semester on the "Mathematical Modelling of the Cardiovascular System". I acknowledge Prof. A.Quarteroni for guidelines dealing with haemodynamics framework and mathematical modelling, C.Prud'homme, Prof. A.T.Patera and K.Veroy (MIT) for reduced basis tools applied to PDEs, Prof. V.Agoshkov (Russian Academy of Sciences) for optimal control framework and adjoint problems in shape design.

\section{REFERENCES}

1. Agoshkov V, Quarteroni A, Rozza G. Shape design approach using perturbation theory for bypass anastomoses. Preprint, 2004.

2. Cole JS, Watterson JK, O'Reilly MGJ. Numerical investigation of the haemodynamics at a patched arterial bypass anastomosis. Medical Engineering and Physics, 2002; 24: 393-401.

3. Lions JL. Optimal Control of Systems Governed by Partial Differential Equations. Springer-Verlag, 1971.

4. Mohammadi B, Pironneau O. Applied Shape Optimization for Fluids. Clarendon Press, Oxford, 2001.

5. Patera AT, Rozza G, Veroy K. Reduced basis methodologies for Stokes equations in parametrized domains. In preparation, 2004

6. Perktold K, Hofer M, Karner G, Trubel W, Schima H. Computer simulation of vascular fluid dynamics and mass transport: optimal design of arterial bypass anastomoses. Proceedings of ECCOMAS 1998, K. Papailion and others Editors, John Wiley and Sons: 484-489.

7. Prud'homme C, Rovas D, Veroy K, Maday Y, Patera AT and Turinici G. Reliable real-time solution of parametrized partial differential equations: reduced-basis output bound methods. J. Fluids Engineering, 2002; 172: 70-80.

8. Prud'homme C, Rovas D, Veroy K and Patera AT. Mathematical and computational framework for reliable real-time solution of parametrized partial differential equations. $M^{2} A N$, Mathematical Modelling and Numerical Analysis, 2002; 36(5): 747-771.

9. Quarteroni A, Rozza G. Optimal control and shape optimization in aorto-coronaric bypass anastomoses. $M^{3} A S$, Mathematical Models and Methods in Applied Sciences, 2003; 13(12): 1801-23.

10. Quarteroni A, Formaggia L. Mathematical modelling and numerical simulation of the cardiovascular system, in Modelling of Living Systems, Handbook of Numerical Analysis Series (P.G. Ciarlet e J.L. Lions Eds), Elsevier, Amsterdam, 2003.

11. Quarteroni A, Tuveri M, Veneziani A. Computational vascular fluid dynamics: problems, models and methods. Computing and Visualization in Science, 2000; 2: 163-197.

12. Quarteroni A, Valli A. Numerical Approximation of Partial Differential Equations. Springer-Verlag, Berlin, 1994.

13. Rozza G. Reduced basis methods for elliptic equations in sub-domains with a posteriori error bounds and adaptivity. HaeMOdel and Mox report 27, 2003, Politecnico di Milano. 